Article (refereed)

Prunet, Patrick; Cairns, Michael T.; Winberg, Svante; Pottinger, Thomas G.. 2008 Functional genomics of stress responses in fish. Reviews in Fisheries Science, 16 (S1). 157-166. doi:10.1080/10641260802341838

Copyright: () Taylor and Francis Group, LLC

This version available at http://nora.nerc.ac.uk/3704/

NERC has developed NORA to enable users to access research outputs wholly or partially funded by NERC. Copyright and other rights for material on this site are retained by the authors and/or other rights owners. Users should read the terms and conditions of use of this material at http://nora.nerc.ac.uk/policies.html\#access

This document is the author's final manuscript version of the journal article, incorporating any revisions agreed during the peer review process. Some differences between this and the publisher's version remain. You are advised to consult the publisher's version if you wish to cite from this article.

www.informaworld.com/ 


\section{Functional genomics of stress responses in fish}

PATRICK PRUNET ${ }^{1 *}$, MICHAEL T. CAIRNS ${ }^{2}$, SVANTE WINBERG $^{3}$, THOMAS G. POTTINGER $^{4}$

${ }^{1}$ INRA-SCRIBE, Fish Physiology of Stress and Adaptation group, IFR 140, campus de Beaulieu, 35042 Rennes Cedex, France.

${ }^{2}$ National Diagnostics Centre, National University of Ireland, Galway, Ireland.

${ }^{3}$ Department of Comparative Physiology, Uppsala University, Uppsala, Sweden.

${ }^{4}$ Centre for Ecology and Hydrology, Lancaster Environment Center, Library Avenue, Bailrigg, Lancaster, LA1 4AP, UK.

\section{* Corresponding author}




\section{ABSTRACT}

Our understanding of the mechanisms underlying stress responses in fish remains fragmentary. However, new insights into these mechanisms, and their biological significance, have been provided by investigation at the transcriptional level. Microarray technology has allowed the unbiased analysis of the transcriptome, providing a potentially system-wide overview of stress responses. In this review, we present recently published transcriptomic studies on stress responses in fish exposed to a range of environmental, xenobiotic, social, and aquacultural stressors. Overall, these studies highlight the complexity of transcript patterns, have identified new genes whose expression is significantly modified after exposure to stressors, and have revealed both common and tissue-specific expression signatures. Some shortcomings can be identified including lack of information on the longer-term compensatory or adaptive phases of the stress response, limitations on gene annotation, and the use of pooled mRNA preparations which masks variation between individuals. Nonetheless, although the functional genomic analysis of stress responses in fish is still in its infancy, rapid growth in the number of studies and continued advances in technology and database content will inevitably lead to a fuller understanding of the processes involved and to the identification of novel stress indicators with diagnostic or predictive value.

Keywords: fish, stress, environmental factors, functional genomics, proteomics 


\section{INTRODUCTION}

There is now a general consensus that stress in teleost fish is a condition in which homeostasis is threatened as a result of the actions of external stimuli which can be defined as stressors. The neuroendocrine, physiological and behavioural alterations that comprise the stress response are considered to be compensatory or adaptative mechanisms that allow the fish to cope with stressors in order to maintain homeostasis and ultimately to survive. Thus, the stress response is essentially beneficial to the fish in the short-term. However, if the intensity of the stressor(s) is overly severe or exposure to the stressor(s) is prolonged, the stress response may lose its adaptative value and become maladaptive and detrimental to the fish's health and well-being (reviewed by Barton and Iwama, 1991; Wendelaar Bonga, 1997; Barton, 2002).

The physiological stress response has been separated into primary and secondary elements: primary responses comprise the initial neuroeondocrine changes including the release of catecholamines by the chromaffin tissue and the activation of the hypothalamic-pituitaryinterrenal axis resulting in the release of corticosteroids; secondary responses are exemplified by the manifold immediate actions of these hormones at blood and tissue levels, which include effects on metabolism, respiration, acid-base status, hydromineral balance, immune function and cellular function. Additionally, tertiary responses, which include adverse alterations at the level of the whole animal and even populations have also been defined and include changes in peformance factors such as growth, disease resistance, and behaviour (Pickering, 1981; Pickering and Pottinger, 1995; Iwama et al., 1997; Mommsen et al., 1999). However, this rather rigid classification does not reflect the complexity and the flexibility of stress responses in fish which may vary according to the duration, intensity and context of the stressor(s). Currently, knowledge about the physiological response of fish to stress is related to the primary elements of the neuroendocrine cascade of the brain-chromaffin and 
hypothalamic-pituitary-interrenal (HPI) axis and their subsequent secondary effects. A limited range of species has been studied in detail and much of the research is focused on important aquaculture species. Furthermore, until recently, few studies evaluated the effects of environmental perturbations related to natural or anthropogenic stressors, particularly waterborne toxic chemicals (Barton, 2002). Due to the intimate contact between fish and their environment and the presence of a large variety of perceptive mechanisms at the level of the integument, these toxic pollutants have profound effects via both direct toxic effects and nonspecific stress responses (Wendelaar Bonga, 1997).

Although stress responses in fish have been extensively studied at the effector level of hormones, receptors, enzymes and metabolites, few studies have examined the molecular mechanisms that underly the physiology of the stress response and our undertsanding of these underpinning mechanisms is fragmentary. While the analysis of candidate genes has provided useful new information, genomic screening approaches using microarray technology have only recently been applied to the study of stress responses in fish. These new screening methods potentially provide a system-wide assessment of stress-induced alterations in gene transcription which should not only generate overviews of the biological pathways and processes involved in the stress response but also provide the means to identify new candidate genes (Cossins et al., 2006). These strategies are based on the assumption that data generated by a genomic approach provides a more comprehensive view of the complexity of the responses to stressors. Initially the objective of the present review was to identify both stressor-specific and stressor-non-specific molecular markers by meta-analysis of published studies. However, it became evident on reviewing the literature that such goal was unrealistic as the stressors employed in published studies are varied in nature or intensity, a range of fish species has been employed and the functional genomic tools used also differ. These inconsistencies between studies are compounded by shortcomings in the quality and extent of 
EST collections employed. In this context, it is inappropriate to carry out meta-analysis of functional genomics data on stress responses in fish and instead the present review will consider the contribution of genomic approaches to understanding stress responses in fish. The effect of abiotic and biotic stressors related to aquaculture practices will be reviewed as will genomic studies of the physiological responses to other environmental challenges in both aquaculture species and model species such as zebrafish (Danio rerio) or medaka (Oryzias latipes).

\section{GENOMIC RESPONSES TO MODEL AQUACULTURE STRESSORS}

Fish in aquaculture are frequently exposed to a range of "unnatural" stressors which are related to rearing practices. These include overcrowding, handling and transport. Study of the effects of such stressors in aquaculture fish species has led to an abundant literature with many studies focused on endocrine responses and metabolic adjustement at the level of tissue and plasma (Mommsen et al., 1999). In most of these studies, exposure to stressors was carried out using standardized protocols which impose acute or chronic handling stress and/or confinement. Activation of the HPI axis is one of the major endocrine responses to such stressors and administration of cortisol has often been used to simulate stress situations and further investigate the physiological consequence of HPI activation (Barton, 2002). The application of microarray technology has allowed the specific modifications of the transcriptome in response to confinement/handling stress in fish to be studied in detail.

At this stage, presenting a comprehensive synthesis of the results of the first genomic studies is difficult as although most of the studies have been carried out in rainbow trout (Oncorhynchus mykiss), the protocols for stress exposure differ between studies and include single or repeated episodes of acute stress with or without recovery, or chronic stress exposure. Moreover, transcriptome analysis was carried out in different tissues in the 
different studies and in only one study was the gene expression profile compared in two tissues. Finally, cortisol administration studies which aim to mimic aspects of the secondary stress responses have so far only been carried out in a limited number of situations, i.e. in vivo on seabream kidney or in vitro on trout hepatocyte cells in primary culture. Despite these limitations it is possible to draw a number of conclusions from transcriptomic analyses.

- As expected, all transcriptomic analysis led to identification of a complex network of genes whose expression is modified after exposure to handling or confinement stress. Thus, when searching for enriched Gene Ontology (GO) functional categories in the list of differentially expressed genes in trout exposed to repeated acute netting stress, Krasnov et al. (2005a) found alterations in functional classes such as binding and transport of metal ions, chaperone and heat shock proteins, cytoskeleton and microtubules and a number of signaling pathways in the brain, whereas in the kidney GO classes such as mitochondrion, extracellular structures and peptidases were highlighted. A similar analysis was also carried out with the head-kidney of trout exposed to a confinement stressor and revealed changes in biological functions such as protein turn-over, energy demand, steroidogenesis, immune response, detoxification, oxygen transport, cellular structure, electron transport chain, signal transduction, and ion transport (Le Borgne G. and P. Prunet. unpublished data). Although no GO analysis was conducted, studies on the effect of an acute stressor on the trout liver transcriptome also indicated significant changes in expression of genes involved in different biological functions: These included inflammation (acute phase proteins; Cairns et al., 2007), gluconeogenesis and glycogenolysis (Wiseman et al., 2007; Momoda et al., 2007), energy metabolism (Wiseman et al., 2007), proteins involved in protein degradation (Wiseman et al., 2007) and the immune response (Wiseman et al., 2007; Momoda et al., 2007). Similar complexity was apparent in trout hepatocytes treated with cortisol in vitro and genes with 
modified expression included those involved in intermediate metabolism, cellular stress response, reproduction and xenobiotic metabolism (Aluru et al., 2007).

- Interestingly, transcriptome analysis in tissue from fish exposed to a confinement or handling stressor revealed the involvement of cellular mechanisms not previously identified in this context. Thus, a transient change in immune-related genes in response to acute stress was observed in trout liver (Wiseman et al., 2007; Momoda et al., 2007; Le Borgne G. and P. Prunet. unpublished data). Exposure to an acute and chronic stressors also led to an increase in haptoglobin expression in trout liver and head-kidney, a prominent acute phase protein which has never previously been associated in fish with non-immunological stressors (Cairns et al., 2007; Le Borgne G. and P. Prunet. unpublished data). Another interesting finding arising from transcriptome analysis is the possible involvement in the stress response of hormones not previously associated with this process in fish, such as insulin-like growth factors, testosterone and insulin in the liver (Wiseman et al., 2007) and in the head-kidney, prolactin, growth hormone and somatolactin (Le Borgne G. and P. Prunet. unpublished data).

- Transcriptome analysis is uniquely suited to the identification of potential new biomarkers of acute and chronic stress. Such information could be found in the transcriptome analysis of acute stress experiments (Krasnov et al., 2005a; Momoda et al., 2007; Wiseman et al., 2007; Sarropoulou et al., 2005) but appears more clearly in the large time-course analysis of confinement stress carried out on trout by Cairns et al. (2007) and Le Le Borgne G. and P. Prunet. (unpublished data). In rainbow trout exposed to an ongoing chronic stressor, and using a sampling protocol which ranged from 2 hours to 21 days of confinement stress, these studies identified genes in the liver and headkidney whose expression showed short-term or long-term modification which offer potential as markers of acute/chronic stress. The potential importance of reliable stress 
markers warrants further investigations aimed at validating the utility of these candidate genes as markers of acute/chronic stress.

Despite the wealth of transcriptomic analysis represented by these studies, we are far from achieving a comprehensive view of the molecular mechanisms involved in the response of fish to the model stressors, handling and confinement. This goal requires further studies. In particular, more accurate statistical analysis should be conducted on large EST collections in order to have a wider view of gene expression changes in target cells. Recent progress in the construction of large EST collections for several aquaculture species and the development of large size oligo microarrays should make such analysis possible in the very near future (see Canario et al., present issue). In addition, an improvement in our understanding of the complexity of the stress response to standardised model stressors such as confinement will also come from transcriptome analysis of target cells in primary culture exposed to corticosteroid or catecholamines, the major endocrine factors which regulate many of the secondary stress responses.

\section{EFFECTS OF AGONISTIC BEHAVIOUR AND SOCIAL STRESS}

The formation of dominance hierarchies due to competition for limited resources, such as food and foraging sites is evident in several teleost species, including salmonids, in the wild as well as in captivity (Yamagishi, 1962; Symons, 1968; Jenkins, 1969; Abbott and Dill, 1989; Metcalfe et al. 1989; Nakano, 1995). Socially subordinate fish experience unreliable access to food, a general lack of control and predictability, and a constant threat of aggression from dominants (Zyan, 1991; Winberg and Lepage, 1998). Thus social subordination is stressful and characterised by elevated levels of plasma cortisol and brain serotonin metabolism together with other indicators of sustained stress. In the wild, social status is a 
major determinant of life history traits, reproductive success and survival in many species (reviewed in Johnsson et al., 2006). In aquaculture regimes, although social hierarchies become less distinct with increasing stocking densities (Jørgensen et al., 1993; Jobling and Baardvik, 1994) growth depensation is a common phenomenon, and fish that grow poorly may do so because they are socially subordinate (Cubitt et al., 2008). In addition to economic issues, the chronic stress experienced by subordinate fish is a serious issue with regard to fish welfare in commercial aquaculture.

Differences in behaviour, hormone levels and neurochemistry associated with social status in various teleosts appear mainly as a result of chronic stress in subordinates (reviewed in Summers and Winberg, 2006). However, knowledge of the molecular mechanisms underlying social stress and complex social behaviors, such as aggression and the formation of dominance hierarchies, is limited. The fact that the social status of an animal might change during its life time suggests that regulation of gene activity is an important mechanisms for the control of agonistic behaviour. The complexity of behavioural traits suggests that they are polygenic and that gene interactions are fundamental. Thus, genomic multigene approaches hold great potential to reveal these complex networks of interrelated mechanisms.

Sneddon et al. (2005) applied a microarray approach to study gene expression profiles in the brain of rainbow trout of divergent social status. The results show that the expression of as many as 1165 genes differed between fish of different social status. Given the link between social rank and food intake, feed conversion ratio and growth rate it is no surprise that the majority of genes found to be differentially expressed in the study by Sneddon et al. (2005) were related to processes like protein turnover and metabolism. However, a number of genes related to stress responses and behaviour which could serve as candidates for future studies were also identified (Sneddon et al., 2005). Aubin-Horth et al. (2007) used a DNA microarray constructed from a African cichlid (Astatotilapia burtoni) brain-specific cDNA library (Renn 
et al., 2004) to study brain gene expression profiles in dominant male and female breeders and subordinate male and female helpers of the African cichlid Neolamprologus pulcher. In this study four genes were found to vary between dominant and subordinate fish independent of sex. One of these genes was arginine vasotocin (AVT), which could be considered a candidate gene as it has been linked to aggressive (dominance, territoriality) and reproductive behaviors in fish and birds and to social affiliation and partner preference in mammals (Goodson and Bass, 2001), whereas the others genes are related to processes like neural growth, ageing and immune reactions, all of which are biological functions not usually associated with social behaviour (Aubin-Horth et al., 2007).

\section{GENOMIC ANALYSIS OF STRESS RESPONSES AND ACCLIMATION TO} ENVIRONMENTAL CHANGES

Alterations in almost all factors related to physical characteristics of the aquatic environment (temperature, salinity, turbidity, $\mathrm{pH}$, oxygen level, xenobiotics.) can result in an integrated stress response in fish if the changes are sudden or sufficiently extreme (Wendelaar Bonga et al., 1997; Barton, 2002). Over the past 30 years, studies of the stress response caused by environmental challenges in fish have focused on the physiological responses for the maintenance of homeostasis and their endocrine regulation. Much less information has been produced on the stress responses that occur at the cellular levels, and the relationships between known molecular mechanisms involved in these stress responses are still poorly understood. In addition to defensive, or immediately adaptive responses that occur at the cellular level in reaction to an environmental challenge, a second type of adaptation cellular response has been described, which restores cellular homeostasis when changes in environmental conditions become permanent (Kültz, 2005). The recently published genomic studies related to environmental stress and acclimation offer new insights into the role of the 
transcriptome and proteome in the regulation of physiological responses to environmental challenge.

\section{Effects of temperature change}

Because they are poikilotherms, fish experience the full effects of fluctuations in environmental temperature. Moreover, many fish species experience large diurnal and seasonal temperature changes which make their physiological responses a favoured subject for studying the mechanisms that underly environmentally induced physiological plasticity (Cossins et al., 2006). Genomic techniques offer the opportunity to identify new mechanisms underpinning these biological processes, and to investigate in greater detail those that are already documented. Although several of the studies described here were designed to investigate molecular mechanisms of acclimation to naturally occurring temperature regimes, the results provide relevant insight into the mechanisms employed by fish exposed to stressful temperature change. A time-course analysis of transcript expression in seven tissues was carried out in carp (Cyprinus carpio) exposed to graded cooling regimes (Gracey et al., 2004; Gracey, 2007) and showed that a large set of genes (3,400 unique cDNA) were affected by cold acclimation. The complexity of the transcriptome responses led the authors to focus on the detection of biological themes within the expression data and to interpret the functional role of the complex changes in gene expression. Thus, by unbiaised profiling of gene ontologies using GO terms, Gracey et al. (2004) were able to visualize the major biological functions corresponding to genes showing significant up- or down-regulation. This study highlighted the most distinctive features of adjustment to cold in each of the nine tissues. For example, this thematic analysis clearly showed the importance of lipid for proper function of the central nervous system and also suggested a specific role for genes involved in protein folding during cold acclimation. Finally, the study revealed a range of genes linked to 
thermotolerance and highlighted the complexity of the molecular responses to temperature acclimation.

Based on the transcriptome analysis (Gracey et al., 2004) which suggested that carp muscle responded to cold acclimation by reductively remodelling its contractile apparatus, McLean et al. (2007) pursued that question and carried out a proteomic analysis of the carp muscle using the same temperature challenge protocol. They showed that chronic exposure of carp to low temperature produced subtle changes in the pattern of soluble proteins most notably with the appearance of a multiple breakdown fragments of the creatine kinase isoforms. This observation is consistent with a cold-induced enhancement of proteolysis and with a remodelling of the cellular creatine kinase expression shown in the previous study.

Another original approach was also developed by Podrabsky and Somero (2004) when analyzing the transcriptome in killifish (Austrofundulus limnaeus) acclimated to 3 constant temperatures ranging between $20^{\circ} \mathrm{C}$ and $37^{\circ} \mathrm{C}$ or exposed to daily fluctuations of temperature between $20^{\circ} \mathrm{C}$ and $37^{\circ} \mathrm{C}$. These situations correspond to natural temperatures to which this fish species is exposed in the environment. Gene expression analysis in liver revealed major differences between these various situations although fewer genes showing significantly different expression were observed in this study compared to that of Gracey et al. (2004). However, numerous new hypotheses related to effects of temperature acclimation on cellular pathways were generated by the study, the most notable being the possibility that the high mobility group B1 (HMGB1) may act as a compensatory modulator of transcription in response to temperature and thus act as a temperature sensor for global gene expression.

Short-term cellular responses to heat stress have also been analyzed by genomic approaches (Buckley et al., 2006): to mimick an ecologically relevant temperature change gobies (Gillichthys mirabilis) were exposed to a thermal regime $\left(18^{\circ} \mathrm{C}\right.$ to $32^{\circ} \mathrm{C}$ over 3 hours, $32^{\circ} \mathrm{C}$ for 2 hours and back to $18^{\circ} \mathrm{C}$ over 3 hours) and gene profile analysis was carried out in gill 
and white muscle. In common with previous studies, such analysis revealed both expected and unexpected changes in gene expression. A GO analysis allowed the authors to groups changing genes within 14 different biological processes covering many cellular mechanisms. Among the most inducible set of genes were those of the molecular chaperones including the heat shock proteins. Interestingly, this study also examined the correlation between gene expression and the corresponding protein levels measured in gill or muscle. In every case, an induction in mRNA production was correlated with an increase in protein concentration, although relative timing and magnitude of transcript levels versus that of protein varied from gene to gene. Finally, it is interesting to note that this study revealed fewer genes with a significant response to temperature changes than the study of Gracey et al. (2004). Buckley et al. suggested that the difference in findings may stem from the fact that their study corresponded to an acute temperature stressor occuring over a few hours whereas Gracey et al. (2004) analyzed an acclimation process which developed over the course of 22 days. These studies probably correspond to the two-stage stress response defined by Kültz (2005), i.e. for the study by Buckley et al. (2006), an early reaction to environmmental stress, also referred to as the 'cellular stress response' and for the study by Gracey et al. (2004), a longer term response also called the 'cellular homeostatic response'. The changes in gene expression that underpin the compensatory adjustments during a longer-term acclimation may be more extensive than those associated with the immediate damage prevention which occurs during an early reaction to stressors.

At this stage, it is still difficult to establish a complete overview of the molecular mechanisms which underpin the response to temperature stress and acclimation although significant progress has recently been made. A transcriptomic analysis alone may not easily identify the key biological process involved in temperature effects and complementary functional analysis is also required. Morever, differences between the conditions under which related studies 
were undertaken also introduces uncertainty. For example, whereas gene expression profiling in zebrafish clearly identifies oxidative stress as a principal biological theme affected by a year-long $10^{\circ} \mathrm{C}$ reduction in water temperature, oxidative damage as assessed by three widely used molecular markers was not modified by temperature reduction (Malek et al., 2004). It is therefore unclear whether the different results in obtained in response to changes in temperature reflect the different experimental conditions, or suggests a more complex regulation of the process than anticipated.

\section{Effects of hypoxia}

Hypoxia is an important stress factor for all fish species and was among the first stressors to be analyzed using a genomic approach. An extended transcriptome analysis carried out in the liver and muscle of adult gobies (Gillichthys mirabilis; Gracey et al., 2001) exposed to hypoxia during 6 days led the authors to derive a coherent view of the mechanisms promoting survival under hypoxic conditions: first, a rapid shut down of the major energy-requiring processes, such as protein synthesis and locomotion at the level of muscle, followed by a strong induction of genes involved in anaerobic ATP production and gluconeogenesis at the level of the liver, associated with suppression of cell-growth and proliferation and with a down-regulation of genes encoding protein of the translation machinery. A similar study carried out in zebrafish embryos exposed to hypoxia for 24 hours also revealed downregulation of genes involved in similar cellular functions such as 'energy consumption', 'contractile, extracellular matrix and cytosqueleton proteins', 'protein translation machinery', and 'cell division' (Ton et al., 2003). In medaka exposed to short term hypoxia (4days), Ju et al. (2007a) carried out a microarray analysis of gene expressions in various target tissues (gill, liver, brain) and suggested that two biological functions, ubiqutin-proteasome and phosphatidylinositol pathways were significantly dysregulated. Investigation of gene expression change in zebrafish exposed to long-term hypoxia led van der Meer et al. (2005) to 
suggest that induction of genes for lysosomal lipid trafficking and degradation is a long-term adaptative mechanism to hypoxia.

Beyond an overview of the hypoxia-induced mechanism; such gene profiling analysis in tissue exposed to hypoxia also led to the unexpected discovery of myoglobin expression in non-muscle tissues (Fraser et al., 2006). The functional genomic approach adopted in this study consistently showed an over-expression of different myoglobins in brain, liver and gill of carp exposed to hypoxia for 8 days. Further protein biochemistry analysis allowed the authors to confirm the presence of a myoglobin protein in the liver, the level of which increased following hypoxia exposure. The role of hypoxia-induced myoglobin in the liver may be to facilitate oxygen delivery to this tissue and thus may account in part for the profound hypoxia tolerance exhibited by carp. Moreover, a new brain-specific isoform of myoglobin was also isolated but transcript levels were unaffected by hypoxia. This is a pertinent example of how large-scale expression screening can lead to the discovery of unexpected gene functionality (Gracey, 2007).

\section{Effects of salinity change}

Alterations in salinity constitute a classic example of the stressor as a threat to homeostasis and the ability of euryhaline fish to tolerate variation in salinity without compromising biological processes has generated a large literature on the osmoregulatory mechanisms which underpin this ability. Based on an hypothesis-driven approach related to the roles of specific candidate genes or proteins, numerous studies have identified major transporters involved in freshwater or seawater osmoregulation (see reviews by Evans et al., 2005; Marshall, 2002; Hoffman et al., 2007; Hwang and Lee, 2007). However, several genomic approaches have recently been developed in order to provide new information on the molecular mechanisms involved in response to salinity changes. Thus, using suppression subtractive hybridisation (SSH), Boutet et al. (2006) have identified 136 different cDNAs corresponding to genes 
involved in several major cellular physiological functions. From these potential candidate genes, they confirmed by real-time PCR the regulation by salinity of several genes involved in osmoregulatory mechanism $\left(\mathrm{Na}^{+} \mathrm{K}^{+}\right.$-ATPase, carbonic anhydrase, angiotensin-converting enzyme, claudin-3), cell-cycle regulation (secretogranin) or the immune system (nephrosin) in gill and intestine.

A more extensive analysis of the transcriptome of eel transferred from freshwater to seawater was carried out by Kalujnaia et al. (2007) using microarray technology. Gene expression profiling in osmoregulatory tissues (gill, kidney, intestine) and in brain provided 229 different cDNAs of which 95 represented the sequences of known genes. These genes were classified into eight different functional groups, including cellular processes such as cell protection and immune system, energy metabolism, detoxification, transport function, structural proteins and regulation of gene transcription and translation. This study confirmed the large range of genes and cellular functions involved in acclimation to seawater, some of them already implicated in this process but many others requiring further characterization in relation to salinity acclimation.

Using high-throughput transcriptomic and proteomic approaches, Kültz et al. (2007) attempted to identify the key molecular components which are associated with osmoregulatory function in fish. Moreover, they also applied bioinformatic analysis to generate models that incorporated biological functions, cellular pathways and molecular functions associated with osmotic stress responses. Using SSH with the gills from tilapia (Oreochromis mossambicus) transferred to seawater, Fiol et al. (2006a) isolated 22 full-length cDNA and were able to identify most of the genes. Kinetic analysis of their change in expression during salinity stress indicated that most of these genes show rapid and transient increase in mRNA abundance. Such rapid changes during hyperosomotic stress indicated that these genes have the characteristics of immediate-early genes (IEG), as originally defined in 
neuronal tissue (Lanahan and Worley, 1998). Such genes are key regulators during environmental adaptation and control the transcriptional regulation of effector genes necessary for adaptation (Kültz et al., 2007). Gene ontology analysis of the SSH genes indicated that the cellular functions that are targeted during osmotic stress in tilapia gill are apoptosis, cell cycle regulation, energy metabolism, organic osmolyte accumulation, lipid transport and membrane protection, modulation of actin-based cytoskeleton dynamics, control of mRNA and protein stability (Fiol et al., 2006a). Studies on the immediate-early gene network involved in tilapia osmoregulation were extended by protein quantification using specific antibodies (Fiol and Kültz, 2005) and confirmed the induction of osmotic stress transcription factor 1 (OSTF1) protein in tilapia gill cells after transfer from freshwater to seawater. Thus, these genomic studies generated information about the molecular mechanisms mobilized by the fish in response to an increase in salinity and which constitute the molecular substrate for the early stress response to salinity.

\section{Effects of water-borne contaminants}

Water pollution arising from anthropogenic activities (e.g. low water $\mathrm{pH}$, heavy metals, organic chemicals) can have important effects on the physiology of fish and, in addition to direct toxic cellular effects, may elicit an integrated "non-specific" stress response. The high sensitivity to environmental pollution that is exhibited by fish is a characteristic of aquatic animals and is related to the intimate contact of respiratory surfaces with the medium together with the large variety of perceptive mechanisms present in the fish integument (including light, mechano-, temperature, electro-, and chemo-receptors). Thus, exposure to water-borne contaminants leads to an increase in stress-related hormone levels and also results in damage at the level of the gill and skin (Wendelaar Bonga, 1997). Although many studies have been devoted to the effects of pollutants on various physiological parameters in fish, in many cases their precise mechanisms of action remain to be clarified. In mammals, gene expression is a 
sensitive indicator of toxicant effects at the cellular level and this approach (termed toxicogenomics) has been widely and convincingly used to study the mechanism of action of toxicants and to identify a genomic signature for the identification of toxic contaminants (Lettieri, 2006). Studies of the effects of environmental toxicants on gene expression using genomic approaches represent a powerful tool to diagnose chemical stressors in the natural environment and to analyse the mechanism of action of such pollutants. The use of transcriptomic and proteomic techniques in ecotoxicology is rather new and offers several potential applications, such as the study of the mode of action, the assessment of exposure to given environmental stressors, the identification of particularly sensitive subsets of ecological populations, and the discovery of new biomarkers of exposure (Ju et al., 2007b; Calzolai et al., 2007).

The first genomic studies developed in the field of ecotoxicology were used to isolate a limited number of genes related to environmental stress or pollutants and then analyze their expression in fish using array technology. Thus, using 110 fragments of stress-related genes from flounder (Platichthys flesus), Williams et al. (2003) compared hepatic expression of these genes in fish reared in polluted and relatively non-polluted estuaries. In another study, in the context of endocrine disruptive chemicals in the aquatic environment, identification of estrogen responsive genes in zebrafish and sheepshead minnow (Cyprinodon variegatus) was carried out and confirmed that estrogenic effects of various compounds can be observed even at low concentrations (Larkin et al., 2002; Hoyt et al., 2003). Based on their first study, Larkin et al. (2003) created a gene array containing 30 genes from sheepshead minnow that were previously identified as estrogen-responsive and used the system to screen endocrinedisrupting compounds that mimic estrogens. A similar approach was also employed by Blum et al. (2004) to identify androgen-related effects in largemouth bass (Micropterus salmoides). Association between gene expression analysis and a standardized embryo toxicity assay using 
zebrafish (DarT test) has been used to test effects of 3,4-dichloroaniline toxicity (Voelker et al., 2007). In addition to molecular information suggesting the involvement of an aryl hydrocarbon receptor-mediated response, this approach gave differential expression responses at doses ten times below the concentration that elicited visible toxic effects.

Other research groups have recently been using functional genomic technology both to (i) identify novel genes to serve as biomarkers and (ii) to understand the molecular mechanisms responding to toxicity. Thus, using a trout microarray comprising 1273 different genes, transcriptome analysis of whole rainbow trout fry exposed to sublethal ranges of $\beta$ naphthoflavone, cadmium, carbon tetrachloride or pyrene indicated increased expression of genes involved in mitochondrial activities, metabolism of metal ions and protein biosynthesis whereas genes related to the immune system and stress response, signal transducion and nucleotide metabolism were down-regulated (Koskinen et al., 2004). A similar study was carried out on the liver and kidney of juvenile trout exposed to carbon tetrachloride as a model hepatotoxic solvent and to pyrene, a model polycyclic aromatic hydrocarbon (Krasnov et al., 2005b). This study, not only confirmed up-regulation of several classical markers of stress exposure and toxicity (e.g. HSP90, metallothionein) in both tissues, but also revealed increased expression of genes encoding mitochondrial proteins involved in oxidative phosphorylation, in common with the results from trout fry. Moreover, the most disruptive effects associated with exposure to pyrene or carbon tetrachloride was the down-regulation of genes implicated in hematopoiesis and the development of immune cells, an observation also reported in trout fry (Koskrinen et al., 2004). Analysis of expression profiles of liver and kidney genes led the authors to suggest several biological processes were involved in the reactions to toxicity of these compounds. That functional genomic information represents an interesting source of genes which could be used as biomarkers of xenobiotic effects but a 
clear picture of the molecular mechanisms which have been disrupted after xenobiotic exposure still await validation.

Using a large collection of zebrafish oligoarrays representing $\sim 15,000$ different genes, Lam et al. (2006) have carried out a comprehensive analysis of the transcriptome kinetics of zebrafish liver exposed to arsenic over a $96 \mathrm{~h}$ period. This study identified global transcriptional changes and biological networks involved in arsenic-induced responses in the liver. Many differentially expressed genes were found to be involved in various biological functions suggesting that damage to DNA and protein together with oxidative stress are the major cellular injuries caused by arsenic exposure. Interestingly, these injuries were also evident from a histopathologic study of arsenic-exposed liver and corroborated changes of expression for genes associated with cytoskeletal organization and cell adhesion. These results, which are comparable to findings reported for mammals, led the authors to propose a model for the arsenic-induced adaptive response of the zebrafish liver and to suggest zebrafish liver as a toxicogenomic model for investigating arsenic toxicity.

Cadmium in the aquatic environment is a contaminant of major concern and many studies have examined its toxicity in fish. Using a custom 500-clone microarray, Sheader et al. (2006) identified changes in several genes related to oxidative stress in flounder exposed to cadmium. A more comprehensive study on the pattern of gene expression in Cd-exposed fish was carried out by Reynders et al. (2006) in carp liver. Various doses of waterborne and dietary cadmium were used in acute and subchronic exposure experiments. Expression analysis of $\sim 650$ liver genes showed that the molecular impact of cadmium exposure is highly dynamic with time and a differential response in gene expression was observed at low compared to high $\mathrm{Cd}$ concentrations. At low $\mathrm{Cd}$ concentrations genes related to energy and lipid metabolism were modified whereas a more general stress response was demonstrated at a high $\mathrm{Cd}$ concentration. 
In conclusion, these recent studies which have applied functional genomic methodology in aquatic toxicology indicate that rapid progress has been made and that the initial promise of these approaches has been at least partially fulfilled. Further application of these techniques should have continued impact on biological discovery in aquatic toxicogenomics.

\section{GENERAL CONCLUSION}

This overview of recent studies on functional genomic analysis of stress responses in fish has clearly indicated that, although application of these methodologies is presently in its infancy, many novel findings have been reported and on-going research in this field should lead to considerable new knowledge among which will be indicators of stress with valuable diagnostic and/or predictive value. Development of high-throughput methods generates large amounts of information which can be collated at distinct levels of biological organisation and will ultimately lead to new understanding of these complex biological stress responses. However, the capability of such approaches to characterize stress responses will be optimal only if the following limitations can be addressed:

- Although published studies using transcriptome analysis clearly confirm the complexity of stress responses regardless of stressor, such an approach has its limitations, particularly with regards to the fact that mRNA abundance does not equate to protein function. It is important to consider other non-exclusive strategies for investigating the response of fish to stress, such as proteomic, genomic mutant screens or systems biology approaches (Feder and Walser, 2005).

- Many published studies represent relatively superficial investigations of the problems. Several reasons explain this situation: the limited size of the available collection of ESTs used for microarray construction; limited time-course studies; limits to the number of 
experimental situations and tissues analyzed; limited annotation of the isolated fish EST. The development of new genomic and bioinformatic tools (see Canario et al. this issue) should to a large extent address most of these questions.

- Most published studies have (through necessity) used fish for which no information on genetic pedigree is available. Study of cardiac metabolism and its associated transcriptome in Fundulus heteroclitus clearly indicated that there are individual variations in mRNA expression which explain substrate-specific cardiac metabolism (Crawford and Oleksiak, 2007; Oleksiak et al., 2005). As a consequence, this implies that analysis of a limited number of inbred individuals could give rise to misleading conclusions. Consequently, it would not be surprising if similar stress situations gave rise to significantly different gene expression profile outcomes depending on the developmental stage or the strain of fish used in the experimental protocol (Momoda et al., 2007). This is an important issue which should not be overlooked in our studies on the genomics of stress in fish.

- Finally, a precise definition of the experimental parameters, including environmental conditions, feeding regimes, sex and size of the fish, which apply to the stressor exposure experiments is probably key to the successful analysis and interpretation of microarray data related to stress exposure. GO and meta-analysis of different experiments will be interesting approaches to clarify such issues.

\section{ACKNOWLEDGEMENTS}

This review has been carried out within the project "AQUAFUNC" (EU-SSA-022685) with financial support from the Commission of the European Communities and summarizes the results of studies largely funded by the Commission of the European Communities. We wish 
particularly to refer to two projects, Stressgenes (Q5RS-2001-02211) and Aquafrist (FP6513692).

\section{REFERENCES}

Abbott, J. C. and L. M. Dill. The relative growth of dominant and subordinate juvenile steelhead trout (Salmo gairdneri) fed equal rations. Behaviour, 108: 104-113 (1989).

Aluru, N. and M. M. Vijayan. Hepatic transcriptome response to glucocorticoid receptor activation in rainbow trout. Physiol. Genomics, 31: 489-491 (2007).

Aubin-Horth, N., J.K. Desjardins, Y. M. Martei, S. Balshine and H. A. Hofmann,. Masculinized dominant females in a cooperatively breeding species. Mol. Ecol., 16: 13491358 (2007).

Barton, B. A. Stress in fishes: A diversity of responses within particular reference to changes in circulating corticosteroids. Integ. Comp. Biol., 42: 517-525 (2002).

Barton, B. A. and G. K. Iwama. Physiological changes in fish from stress in aquaculture with emphasis on the response and effects of corticosteroids. Ann. Rev. Fish Dis., 1: 3-26 (1991). Blum, J. L., I. Knoebl, P. Larkin, K. J. Kroll and N. D. Denslow. Use of suppressive subtractive hybridization and cDNA arrays to discover patterns of altered gene expression in the liver of dihydrotestosterone and 11-ketotestosterone exposed adult male largemouth bass (Micropterus salmoides). Mar. Environ. Res., 58: 565-569 (2004).

Boutet, I., C. L. Long Ky and F. Bonhomme. A transcriptomic approach of salinity response in the euryhaline teleost, Dicentrarchus labrax. Gene, 379: 40-50 (2006).

Buckley, B. A., A. Y. Gracey and G. N. Somero. The cellular response to heat stress in the goby Gillichthys mirabilis: A cDNA microarray and protein-level analysis. J. Exp. Biol., 209: 2660-2677 (2006).

Cairns, M. T., M. C. Johnson, A. T. Talbot, J. K. Pemmasani, R. E. McNeill, B. Houeix, A. Sangrador-Vegas and T. G. Pottinger. A cDNA microarray assessment of gene expression in 
the liver of rainbow trout (Oncorhynchus mykiss) in response to a handling and confinement stressor. Comp. Biochem. Physiol. Part D, 3: 51-66 (2008).

Calzolai, L., W. Ansorge, E. Calabrese, N. Denslow, P. Part and T. Lettieri. Transcriptomics and proteomics: Application to ecotoxicology. Comp. Biochem. Physiol. Part D, 2: 245-249 (2007).

Cossins, A., J. Fraser, M. Hughes and A. Gracey. Post-genomic approaches to understanding the mechanisms of environmentally induced phenotypic plasticity. J. Exp. Biol., 209: 23282336 (2006).

Crawford, D. L. and M. F. Oleksiak. The biological importance of measuring individual variation. J. Exp. Biol., 210: 1613-1621 (2007).

Cubitt, K. F., S. Winberg, F.A, Huntingford, S. Kadri, V. O. Crampton and Ø. Øverli. Social hierarchies, growth and brain serotonin metabolism in Atlantic salmon (Salmo salar) kept under commercial rearing conditions. Physiol Behav., 94: 529-35 (2008).

Evans, D. H., P. M. Piermarini and K. P. Choe. The multifunctional fish gill: Dominant site of gas exchange, osmoregulation, acid-base regulation and excretion of nitrogenous waste. Physiol. Rev., 85: 97-177 (2005).

Feder, M. E., and J. C. Walser. The biological limitations of transcriptomics in elucidating stress and stress responses. J. Evol. Biol., 18: 901-910 (2005).

Fiol, D. F. and D. Kültz. Rapid hyperosmotic coinduction of two tilapia (Oreochromis mossambicus) transcription factors in gill cells. Proc. Natl. Acad. Sci. USA, 102: 927-932 (2005).

Fiol, D. F., S. Y. Chan and D. Kültz. Identification and pathway analysis of immediate hyperosmotic stress responsive molecular mechanisms in tilapia (Oreochromis mossambicus) gill. Comp. Biochem. Physiol. Part D, 1: 344-356 (2006). 
Fraser, J., L. V. de Mello, D. Ward, H. H. Rees, D. R. Williams, Y. Fang, A. Brass, A. Y. Gracey and A. R. Cossins. Hypoxia-inducible myoglobin expression in nonmuscle tissues. Proc. Natl. Acad. Sci. USA, 103: 2977-2981 (2006).

Goodson, J. L. and A. H. Bass. Social behavior and related anatomical characteristics of vasotocin/vasopressin systems in vertebrates. Brain Res. Brain Res.Rev., 35: 246-265 (2001).

Gracey, A. Y. Interpreting physiological responses to environmental change through gene expression profiling. J. Exp. Biol., 210: 1584-1592 (2007).

Gracey, A. Y., E. J. Fraser, W. Li, Y. Fang, R. R. Taylor, J. Rogers, A. Brass and A. R. Cossins. Coping with cold: An integrative, multitissue analysis of the transcriptome of a poikilothermic vertebrate. Proc. Natl. Acad. Sci. USA, 101: 16970-16975 (2004).

Gracey, A. Y., J. V. Troll and G. N. Somero. Hypoxia-induced gene expression profiling in the euryoxic fish Gillichthys mirabilis. Proc. Natl. Acad. Sci. USA, 98: 1993-1998 (2001).

Hoffmann, E. K., T. Schettino and W. S. Marshall. The role of volume-sensitive ion transport systems in regulation of epithelial transport. Comp. Biochem. Physiol. Part A, 148: 29-43 (2007).

Hoyt, P. R., M. J. Doktycz, K. L. Beattie and M. S. Jr Greeley. DNA microarrays detect 4nonylphenol-induced alterations in gene expression during zebrafish early development. Ecotoxicology, 12: 469-474 (2003).

Hwang, P. P. and T. H. Lee. New insights into fish ion regulation and mitochondrion-rich cells. Comp. Biochem. Physiol. Part A, 148: 479-497 (2007).

Iwama, G. K., A. D. Pickering, J. P. Sumpter and C. B. Schreck (ed.). In: Fish stress and health in aquaculture. Soc. Exp. Biol. Sem. Ser. 62. Cambridge Univ. Press, Cambridge, U.K. (1997). 
Jenkins, T. M. Jr. Social structure, position choice and micro-distribution of two trout species (Salmo trutta and Salmo gairdneri) resident in mountain streams. Anim. Behav. Monogr., 2: $56-123(1969)$

Jobling, M. And B.M. Baardvik. The influence of environmental manipulations on inter- and intra-individual variation in food acquisition and growth performance od Artic charr, Salvenius alpinus. J. Fish Biol. 44: 1069-1087 (1994).

Johnsson, J., S. Winberg and K. A. Sloman. Social interactions, pp 151-196. In: Behaviour and Physiology of Fish, Vol. 24 of the Fish Physiology Series (Sloman K. A., R.W. Wilson and S. Balshine, Eds.). San Diego: Elsevier (2006).

Jorgensen, E.H. and M. Jobling. The effect of exercise on growth, food utilisation and osmoregulatory capacity of juvenile Atlantic salmon, Salmo salar. Aquaculture 116: 233-246 (1993).

Ju, Z., Wells, M.C., Heater, S.J. and R.B. Walker. Multiple tissue gene expression analyses in Japanese medaka (Oryzias latipes) exposed to hypoxia. Comp. Biochem. Physiol. Part C 145: 134-144 (2007a).

Ju, Z., Wells, M.C., and R.B. Walker. DNA microarray technology in toxicogenomics of aquatic models: Methods and applucations. Comp. Biochem. Physiol. Part C, 145: 5-14 (2007b).

Kalujnaia, S., McWilliams, I.S., Zaguinaiko, V.A., Feilen, A.L., Nicholson, J., Hazon, N., Cutler, C.P. and G. Cramb. A trasncriptomic appraoch to teh study of osmoregulation in European eel Anguilla anguilla. Physiol. Genomics 31: 385-401 (2007).

Koskinen, H., P. Pehkonen, E. Vehniäinen, A. Krasnov, C. Rexroad, S. Afanasyev, H. Mölsa, and A. Oikari. Response of rainbow trout transcriptome to model chemical contaminants. Biochem. Biophys. Res. Commun., 320: 745-753 (2004). 
Krasnov, A., H. Koskinen, P. Pehkonen, C. E. Rexroad III, S. Afanasyev and H. Mölsä. Gene expression in the brain and kidney of rainbow trout in response to handling stress. $B M C$ Genomics, 6: 1-11 (2005a).

Krasnov, A., H. Koskinen, C. Rexroad, S. Afanasyev, H. Mölsä and A. Oikari. Transcriptome responses to carbon tetrachloride and pyrene in the kidney and liver of juvenile rainbow trout (Oncorhynchus mykiss). Aquat. Toxicol., 74: 70-81 (2005b).

Kültz, D. Molecular and evolutionary basis of the cellular stress response. Annu. Rev. Physiol. 67: 225-257 (2005).

Kültz, D., D. Fiol, N. Valkova, S. Gomez-Jimenez, S. Y. Chan and J. Lee. Functional genomics and proteomics of the cellular osmotic stress response in 'non-model' organisms. $J$. Exp. Biol., 210: 1593-1601 (2007).

Lam, S. H., C. L. Winata, Y. Tong, S. Korzh, W. S. Lim, V. Korzh, J. Spitsbergen, S. Mathavan, L. D. Miller, E. T. Liu and Z. Gong. Transcriptome kinetics of arsenic-induced adaptive response in zebrafish liver. Physiol. Genomics, 27: 351-361 (2006).

Lanahan, A. and P. Worley. Immediate-early genes and synaptic function. Neurobiol. Learn. Mem., 70: 37-43 (1998).

Larkin, P., L. C. Folmar, M. J. Hemmer, A. J. Poston, H. S. Lee and N. D. Denslow. Array technology as a tool to monitor exposure of fish to xenoestrogens. Mar. Environ. Res., 54: 395-399 (2002).

Larkin, P., L. C. Folmar, M. J. Hemmer, A. J. Poston and N. D. Denslow. Expression profiling of estrogenic compounds using a sheepshead minnow cDNA microarray. EHP Toxicogenomics.,. 111: 29-36 (2003).

Lettieri, T. Recent application of DNA microarray technology to toxicology and ecotoxicology. Environ. Health Perspect., 114: 4-9 (2006). 
Malek, R. L., H. Sajadi, J. Abraham, M. A. Grundy and G. S. Gerhard. The effects of temperature reduction on gene expression and oxidative stress in skeletal muscle from adult zebrafish. Comp. Biochem. Physiol. Part C, 138: 363-373 (2004).

Marshall, W. S. Na+, Cl-, $\mathrm{Ca}++$ and $\mathrm{Zn}++$ transport in fish gills: retrospective review and prospective synthesis. J. Exp. Zool., 293: 264-283 (2002).

McLean, L., I. S. Young, M. K. Doherty, D. H. Robertson, A. R. Cossins, A. Y. Gracey, R. J. Beynon and P. D. Whitfield. Global cooling: Cold acclimation and the expression of soluble proteins in carp skeletal muscle. Proteomics, 7: 2667-2681 (2007).

Metcalfe, N. B., F. A. Huntingford, W. D. Graham, J. E. Thorpe. Early social status and the development of life history strategies in Atlantic salmon. Proc. R. Soc. Lond. Biol. Sci., 236: 7-19 (1989).

Momoda, T. S., A. R. Schwindt, G. W. Feist, L. Gerwick, C. J. Bayne and C. B. Schreck. Gene expression in the liver of rainbow trout, Oncorhynchus mykiss, during the stress response. Comp. Biochem. Physiol. Part D, 2: 303-315 (2007).

Mommsen, T. P., M. M. Vijayan and T. W. Moon. Cortisol in teleosts: dynamics, mechanisms of action, and metabolic regulation. Rev. Fish Biol. Fish., 9: 211-268 (1999).

Nakano, S. Individual differences in resource use, growth and emigration under the influence of a dominance hierarchy in fluvial red-spotted masu salmon in a natural habitat. J. Anim. Ecol., 64: 75-84 (1995).

Oleksiak, M. F., J. L. Roach and D. L. Crawford. Natural variation in cardiac metabolism and gene expression in Fundulus heteroclitus. Nature Genet., 37: 67-72 (2005).

Pickering, A. D. and T. G. Pottinger. Biochemical effects of stress, pp. 349-379. In: Environmental and ecological biochemistry (P. W. Hochachka and T. P. Mommsen, Eds.). Amsterdam: Elsevier (1995).

Pickering, A. D. (Ed.). Stress and fish. New York: Academic Press (1981). 
Podrabsky, J. E. and G. N. Somero. Changes in gene expression associated with acclimation to constant temperatures and fluctuating daily temperatures in an annual killifish Austrofundulus limnaeus. J. Exp. Biol., 207: 2237-2254 (2004).

Renn, S. C. P., N. Aubin-Horth and H. A. Hofmann.. Biologically meaningful expression profiling across species using heterologous hybridization to a cDNA microarray. BMC Genomics, 5: 42-54 (2004).

Reynders, H., K. Van der Ven, L. N. Moens, V. Van Remortel, W. M. De Coen and R. Blust. Patterns of gene expression in carp liver after exposure to a mixture of waterborne and dietary cadmium using a custom-made microarray. Aquat. Toxicol., 80: 180-193 (2006).

Sarropoulou, E., G. Kotoulas, D. M. Power and R. Geisler. Gene expression profiling of gilthead sea bream during early development and detection of stress-related genes by the application of cDNA microarray technology. Physiol. Genomics, 23: 182-191 (2005).

Schjolden, J. and S. Winberg. Genetically determined variation in stress responsiveness in rainbow trout: Behaviour and neurobiology. Brain Behav. Evol., 70: 227-238 (2007).

Sheader, D. L., T. D. Williams, B. P. Lyons and J. K. Chipman. Oxidative stress response of European flounder (Platichthys flesus) to cadmium determined by a custom cDNA microarray. Mar. Environ. Res., 62: 33-44 (2006).

Sneddon, L. U., J. Margareto and A. R. Cossins. The use of transcriptomics to address questions in behaviour: production of a suppression subtractive hybridisation library from dominance hierarchies of rainbow trout. Physiol. Biochem. Zool., 78: 695-705 (2005).

Summers, C. H. and S. Winberg. Interactions between the neural regulation of stress and aggression. J. Exp. Biol., 209: 4581-4589 (2006).

Symons, P. E. K. Increase in aggression and in strength of the social hierarchy among juvenile Atlantic salmon deprived food. J. Fish Res. Bd. Canada, 25: 2387-2401 (1968). 
Ton, C., T. D. Stalmatiou and C. C. Liew. Gene expression profile of zebrafish exposed to hypoxia during development. Physiol. Genomics., 13: 97-106 (2003).

Voelker, D., Vess, C., Tillman, M., Nagel, R., Otto, G.W., Geisler, R., Schirmer, K. And S. Scholtz. Differential gene expression as a toxicant-sensitve endpoint in zebrafish embryos and larvae. Aquat. Toxicol. 81: 355-364 (2007).

Van den Meer, D.L.M., van den Thillart, G.E.E.J.M., Witte, F., de Bakker, M.A.G., Besser, J., Richardson, M.K., Spaink, H.P., Leito, J.T.D. and C.P. Bagowski. Am. J. Physiol. 289: R1512-R1519 (2005).

Wendelaar Bonga, S. E. The stress response in fish. Physiol. Rev., 77: 591-625 (1997).

Williams, T. D., K. Gensberg, S. D. Minchin and J. K. Chipman. A DNA expression array to detect toxic stress response in European flounder (Platichthys flesus). Aquat. Toxicol., 65: $141-157$ (2003).

Winberg, S. and O. Lepage. Elevation of brain 5-HT activity, POMC expression, and plasma cortisol in socially subordinate rainbow trout. Am J Physiol.., 43: R645-654 (1998).

Wiseman, S., H. Osachoff, E. Bassett, J. Malhora, J. Bruno, G. VanAggelen, T. P. Mommsen and M. M. Vijayan. Gene expression pattern in the liver during recovery from an acute stressor in rainbow trout. Comp. Biochem. Physiol. Part D, 2: 234-244 (2007).

Yamagishi, H. Growth relation in some small experimental populations of rainbow trout fry, Salmo gairdneri Richardson, with special reference to social relations among individuals. Japan J. Ecol., 12: 43-53 (1962).

Zayan, R. The specificity of social stress. Behav. Process, 25: 81-93 (1991). 